\title{
An invasive mole with bilateral kidney metastases: A case report
}

\author{
KUN YAO, YU GAN, YUXIN TANG, JIN TANG, LEYE HE and YINGBO DAI \\ Department of Urology, The Third Xiangya Hospital of Central South University, Changsha, Hunan 410013, P.R. China
}

Received January 26, 2015; Accepted September 14, 2015

DOI: $10.3892 / 01.2015 .3768$

\begin{abstract}
Clinical reports of kidney metastases derived from an invasive mole (IM) are rare. The present study presents the case of a 42-year-old woman who exhibited IM and bilateral kidneys metastases in what may be, to the best of our knowledge, the first report of such a case. Following an induced abortion, the patient initially experienced irregular vaginal bleeding and subsequently presented with left-sided waist and abdominal pain. The patient was admitted to hospital and was clinically diagnosed with gestational trophoblastic neoplasia (GTN), as well as bilateral kidney metastases and a spontaneous rupture of the left kidney. This diagnosis was based on the patient's medical history, which comprised increased levels of human chorionic gonadotropin $\beta$ and aberrant results on computed tomography (CT) scans. The patient subsequently received conservative management to prevent renal damage, and a standard etoposide, methotrexate, actinomycin D, cyclophosphamide and vincristine chemotherapy regimen for GTN was administered. Following eight cycles of chemotherapy, a follow-up CT examination indicated that the damage in the patient's left kidney could not be corrected with conservative treatments, therefore the left kidney was excised and a laparoscopic hysterectomy was performed. The pathological results were consistent with the clinical diagnosis and allowed further classification of the primary tumor as an IM. The present case demonstrated that it is possible for IM to metastasize to the kidney, and furthermore, that this type of metastatic tumor may be fragile and possess the potential to cause spontaneous kidney rupture.
\end{abstract}

\section{Introduction}

An invasive mole (IM), a form of gestational trophoblastic neoplasia (GTN) (1), is a pregnancy-associated disorder, which is caused by a molar pregnancy. It has been reported that $0.5-1 \%$ of partial hydatidiform mole cases and $15-29 \%$ of complete hydatidiform mole cases progressed to become IMs $(2,3)$.

Correspondence to: Professor Yingbo Dai, Department of Urology, The Third Xiangya Hospital of Central South University, 138 Tongzipo Road, Changsha, Hunan 410013, P.R. China E-mail: daiyingbo@126.com

Key words: invasive mole, bilateral kidneys, metastases
Irregular vaginal bleeding is the most common symptom of IM, however, further symptoms caused by bleeding in the metastases, such as hemoptysis and neurological symptoms, may also be detected (2). Myometrial invasion, swollen villi and hyperplastic trophoblast are often considered to be the pathological features of IM (1). The clinical diagnosis of IM relies on medical history, clinical symptoms, laboratory tests and examination using imaging. Pathological results are necessary for confirmed cases. Timely and comprehensive treatment based on chemotherapy can result in a good prognosis (1). In China, the incidence rate of IM following pregnancy is $0.94-1.30 \%(4,5)$. Generally, IM is considered to be a disease with malignant behavior, due to its potential to invade into the myometrium and metastasize to other organs. The most common locations for IM metastases are the vagina, lungs and brain (6,7). Alternative sites of metastases, including the epidural space and bladder, have been rarely reported $(8,9)$. To the best of our knowledge, there have been no cases of IM with subsequent metastasis to the kidney reported in the literature. In the present study, the case of a woman presenting with IM and bilateral kidney metastases is reported.

\section{Case report}

A 42-year-old woman was admitted to the Third Xiangya Hospital of Central South University (Changsha, Hunan, China) on the 18th January 2013, presenting with pain in the left waist and abdomen, which had persisted for 1 week. The patient had undergone an induced abortion 3 months prior to this hospital admittance, and since then had subsequently experienced irregular vaginal bleeding. Therefore, dilation and curettage was performed for incomplete abortion in the primary hospital, however no embryonic tissue was obtained.

A comprehensive examination and evaluation of the patient was performed. A large and pliant mass in the left upper abdomen was palpated during physical examination. The patient exhibited a rapid heart rate $(105 \mathrm{bpm}$; normal range, $60-100 \mathrm{bpm})$ and reduced blood pressure $(85 / 53 \mathrm{mmHg}$; normal range, $90 / 60$ to $140 / 90 \mathrm{mmHg}$ ), combined with markedly decreased levels of hemoglobin $(72 \mathrm{~g} / \mathrm{L}$; normal range, $110-150 \mathrm{~g} / \mathrm{L}$ ), and therefore received supportive treatment in the form of blood transfusions ( $2 \mathrm{U}$ packed red blood cells).

The patient was also administered preventive antibacterial therapy (2.0 g cefamandole nafate, b.i.d. for 2 weeks) and was advised to rest in bed due to the possibility of kidney injury. Laboratory tests revealed markedly elevated levels of human chorionic gonadotropin $\beta$ ( $\beta$-HCG; 462,047 mIU/ml; 

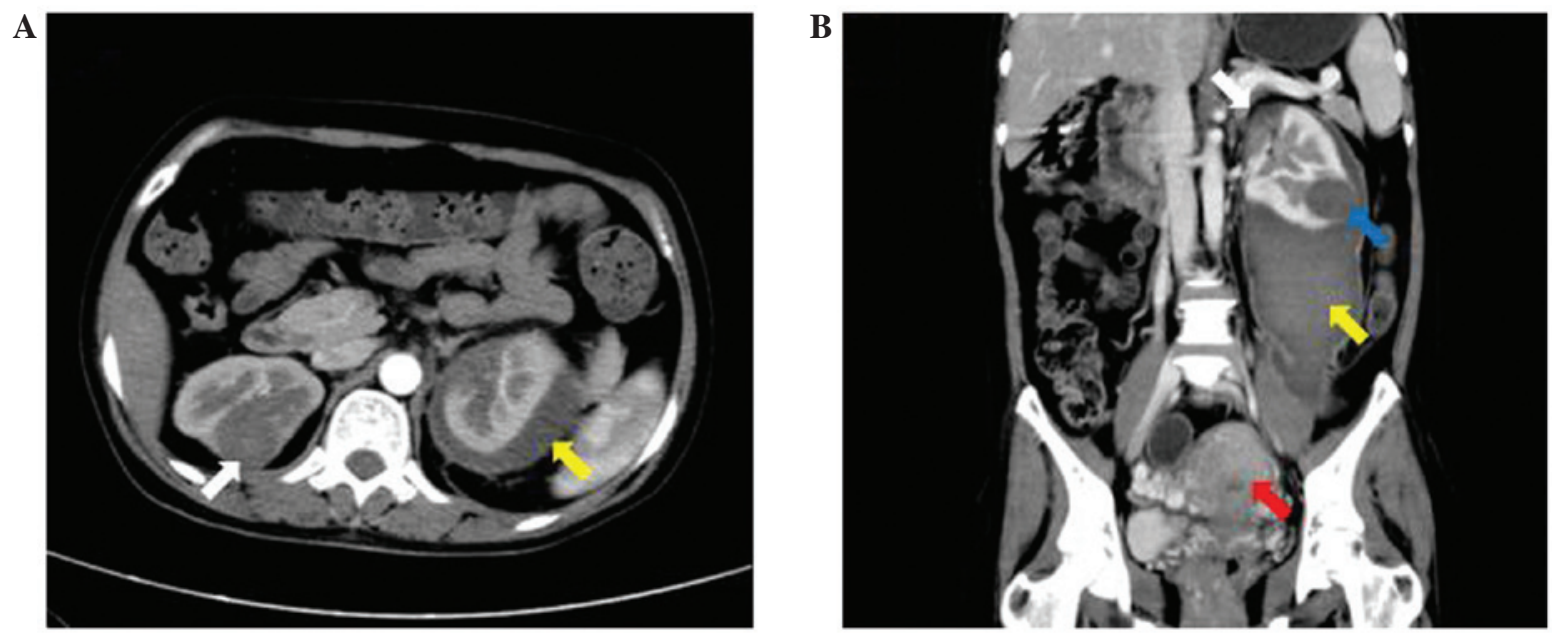

Figure 1. CT scans performed following conservative treatment for renal damage. (A and B) CT scanning revealed masses in the bilateral kidneys (white arrows). Rupture of one of these masses (blue arrow) had caused a retroperitoneal hematoma (yellow arrows). The red arrow indicates the enlarged uterus. CT, computed tomography.
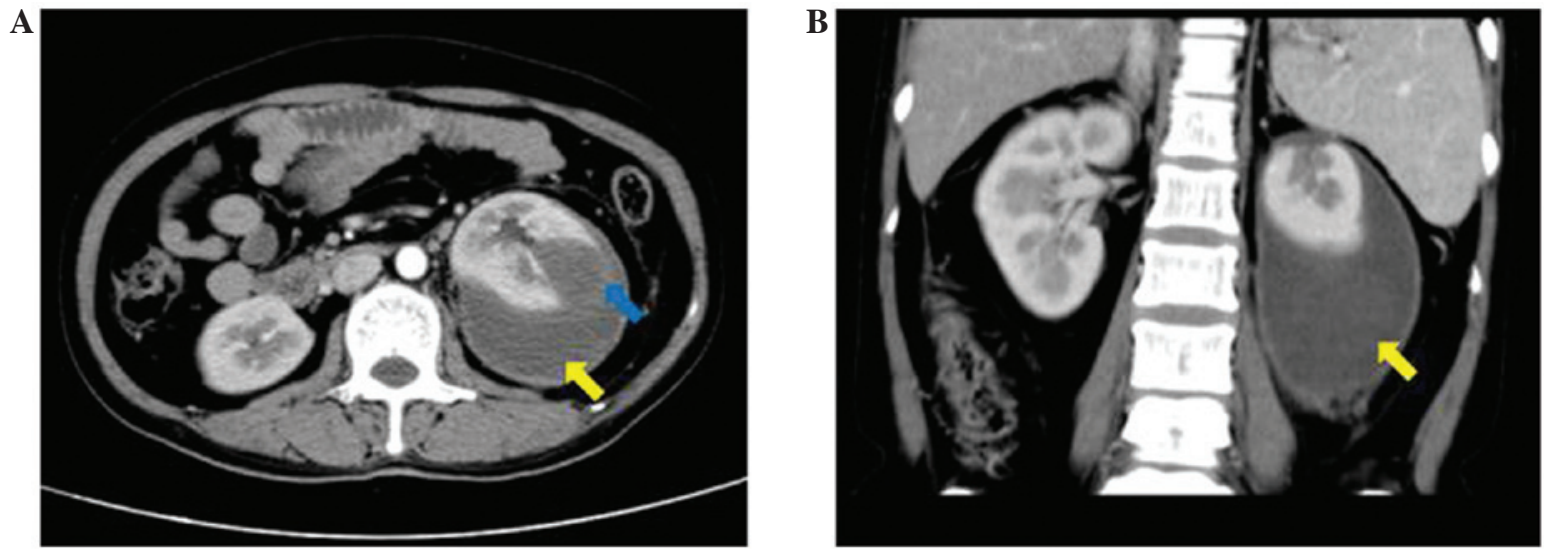

Figure 2. CT scans performed following eight cycles of chemotherapy revealing that the bilateral kidney masses had receded. (A) CT scans revealed that the left kidney had been severely damaged (blue arrow), (A and B) while the retroperitoneal hematoma was observed to have slightly reduced in size, but was still large (yellow arrows). CT, computed tomography.

normal range, $0-10 \mathrm{mIU} / \mathrm{ml}$ ) in the blood, indicating a possible GTN. Computed tomography (CT) identified several bilateral masses in the lungs and kidneys, as well as a large retroperitoneal hematoma, caused by a ruptured mass in the left kidney, and an enlarged uterus. Magnetic resonance imaging (MRI) of the brain also indicated a mass in the right parietal lobe. A second CT scan was performed 1 month later, which revealed that the retroperitoneal hematoma had slightly reduced in size, however, the bilateral masses and enlarged uterus demonstrated no marked alterations compared with the initial CT scan (Fig. 1). The $\beta$-HCG levels in the blood were evaluated every week during the period of conservative treatment (for avoidance of renal damage) and were observed to be continuously increasing. Therefore, the patient was clinically diagnosed with GTN [stage IV; score, 15; according to the FIGO (the International Federation of Gynecology and Obstetrics) staging system and FIGO prognostic scoring system] (10). Furthermore, the masses present in the kidneys, lungs and brain were considered to be metastases from this primary GTN, as the patient possessed no history of previously diagnosed primary tumors at these sites.
The patient was administered standard intravenous EMA/CO chemotherapy every 3 weeks, consisting of:Etoposide $100 \mathrm{mg} / \mathrm{m}^{2}$, methotrexate $300 \mathrm{mg} / \mathrm{m}^{2}$ and actinomycin D $0.5 \mathrm{mg}$ on days 1 and 2 and cyclophosphamide $600 \mathrm{mg} / \mathrm{m}^{2}$, and vincristine $1 \mathrm{mg} / \mathrm{m}^{2}$ (maximum dose of $2 \mathrm{mg}$ ) on day 8 . Simultaneously, a intrathecal injection of methotrexate was administered (15 mg; twice in week $1,10 \mathrm{mg}$ twice in week 2 , every 3 weeks for 4 cycles). Following eight cycles of comprehensive EMA/CO treatment, which lasted for $\sim 4$ months, the $\beta$-HCG levels in the blood had decreased to within the normal range. Furthermore, a second MRI scan of the brain, and a third CT scan, revealed that the masses in the lungs, kidneys and brain had markedly reduced in size. However, the retroperitoneal hematoma remained large and unabsorbed, which increased the risk of infection and aggravated the compression symptoms. In addition, the left kidney was observed to be ruptured (Fig. 2). Therefore, the hematoma was removed and the left kidney was excised during open surgery. It was also recommended that the patient undergo surgery to remove the enlarged uterus, as a mass remained in this area and the patient had no requirement for fertility, therefore a laparoscopic 

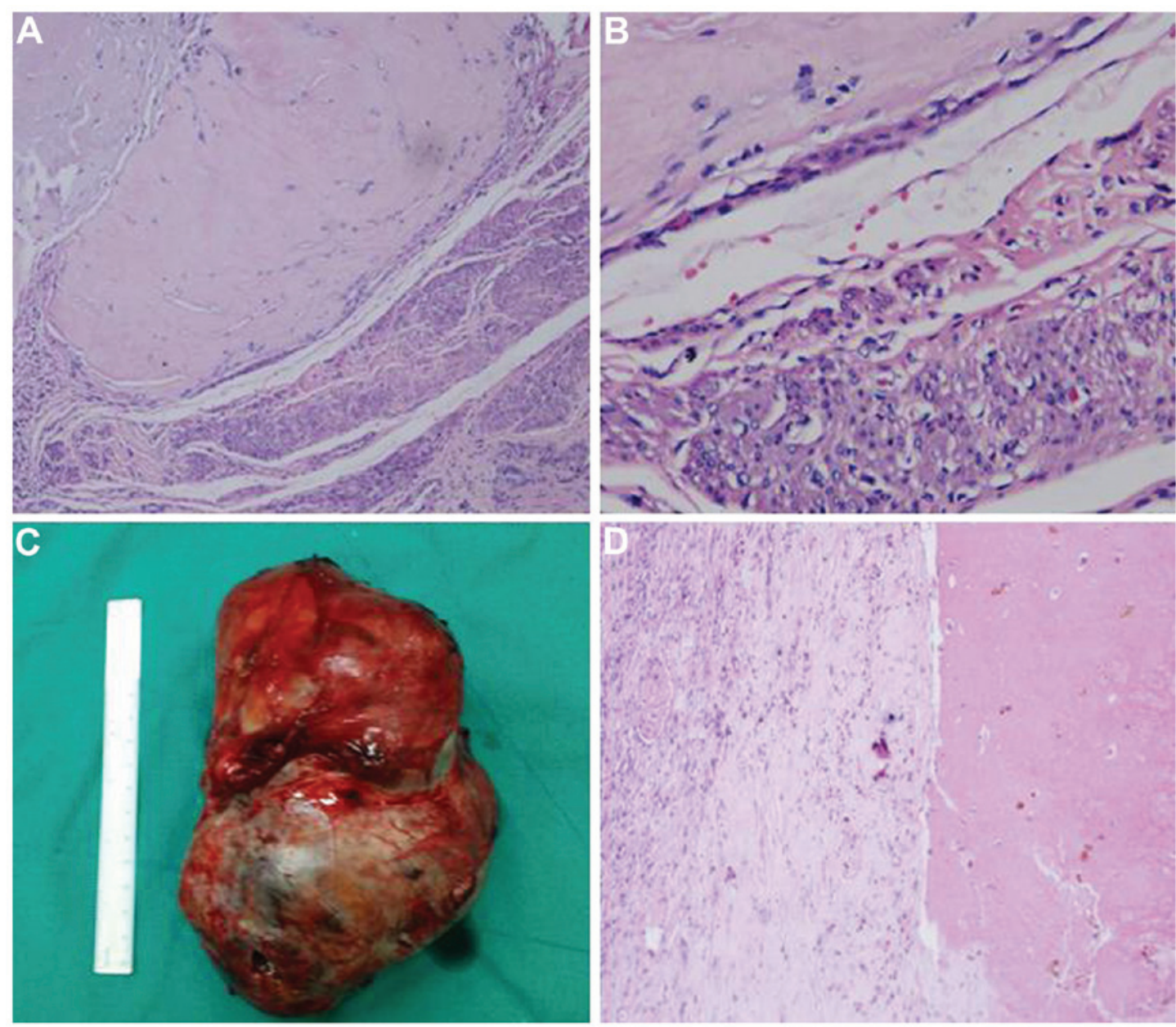

Figure 3. Histopathological examination of the tumor. Histopathological staining of the lesion in the uterus revealed (A) a number of markedly degenerated villi (HE stain; magnification, x10) and (B) trophoblastic cells (HE stain; magnification, x40). (C) The left kidney was excised and a mass with diameter of $3.5 \mathrm{~cm}$ was identified. (D) Histopathological staining also indicated a number of degenerated trophoblastic cells in the lesion and hematoma (HE stain; magnification, x10). HE, hematoxylin and eosin.

hysterectomy was performed. Histological analysis identified degenerated villi and trophoblastic cells in the uterus, as well as metastatic cells in the left kidney, which was consistent with the clinical diagnosis and allowed further classification of the primary tumor as an IM (Fig. 3). The post-operative recovery was uneventful and an additional two cycles of chemotherapy (the same chemotherapy regiments as before operation) were administered. Currently, the patient's blood $\beta$-HCG levels and renal function remain normal, and follow-up of the patient is ongoing.

\section{Discussion}

IM is a common form of GTN. The criteria for the diagnosis of GTN following a hydatidiform mole are as follows: i) A plateau of HCG lasting for four measurements over a period of 3 weeks or longer; ii) an increase in HCG levels following weekly consecutive measurements, over a period of 2 weeks or more; iii) HCG levels remaining elevated for 6 months or longer; and iv) a histological diagnosis of choriocarcinoma (10). Myometrial invasion, swollen villi and hyperplastic trophoblasts are frequently considered to be pathological features of IM, however, the majority of IM cases are diagnosed clinically rather than pathologically (11).
Chemotherapy is a main treatment approach for IM. According to the FIGO staging system (10), patients classified as low risk (stage II-III and score $<7$ ) may receive treatment with a single chemotherapeutic agent, for example methotrexate or actinomycin D $(12,13)$. However, patients classified as high risk (stage IV, or stage II-III and score $\geq 7$ ) are recommended to be treated with combination chemotherapy regimens; EMA/CO has been considered to be the first choice treatment regimen for the last 10 years, and has demonstrated good patient responses and long-term survival rates (14-16). Furthermore, patients exhibiting brain metastases may be administered with systemic chemotherapy, as well as simultaneous administration of one of the three following treatment methods: Whole-brain or stereotactic radiotherapy, intrathecal injection of methotrexate and surgical intervention, for example, craniotomy $(17,18)$. For the treatment of lung metastases, systemic chemotherapy is the typical choice rather than surgery (1).

To the best of our knowledge, a number of cases of choriocarcinoma metastasis in the kidneys have been reported, however, no cases of IM metastasis to the kidneys have been reported $(19,20)$. In the current case, the patient presented with irregular vaginal bleeding following an induced abortion, as well as an elevated $\beta-\mathrm{HCG}$ level for $>3$ months. Furthermore, 
a pathological diagnosis of IM was confirmed following the identification of degenerative villi in the primary lesions of the uterus, and a metastatic tumor was additionally identified in the patient's left kidney.

Based on our experience, we propose that patients exhibiting steady renal metastases should be administered with a systemic chemotherapy regimen and be monitored by strict follow-up appointments. In the event that metastatic tumors rupture and cause subsequent bleeding, patients should be administered a series of conservative treatments for kidney trauma. In addition, essential surgical treatments should be considered when vital signs are unstable or the conservative treatments are deemed to have been ineffective. Conservative and surgical treatments should be accompanied by systemic chemotherapy.

In the present case, the patient was classified as high risk according to FIGO criteria, and was treated with the standard EMA/CO chemotherapeutic regimen, as well as an intrathecal injection of methotrexate. Simultaneously, conservative treatment was administered to remedy the damage to the patient's left kidney. Following eight cycles of this standard chemotherapy, the metastases in the bilateral kidneys had greatly reduced in size, however, the left kidney was observed to be ruptured and was therefore excised, while the right kidney was observed to be intact and was successfully preserved. Following surgery, the patient was subsequently administered an additional two cycles of chemotherapy, in order to consolidate the efficacy of treatment. Currently, this integrated treatment has proven to be effective.

In conclusion, IM metastasis to the kidneys is rarely reported. This may be due to the lack of pathological diagnosis performed on the majority of patients exhibiting GTN. Nevertheless, the present case demonstrated that metastasis of IM to the kidney is possible, and furthermore, that these metastatic tumors may be fragile and possess the potential to cause spontaneous kidney rupture.

\section{Acknowledgements}

The present study was supported by the Hunan Provincial Natural Science Foundation of China (grant no. 14JJ3044) and the Science Foundation of Health and Family Planning Commission of Hunan Province (grant no. B2012-032)

\section{References}

1. Seckl MJ, Sebire NJ and Berkowitz RS: Gestational trophoblastic disease. Lancet 376: 717-729, 2010.

2. Loukovaara M, Pukkala E, Lehtovirta P and Leminen A: Epidemiology of hydatidiform mole in Finland, 1975 to 2001. Eur J Gynaecol Oncol 26: 207-208, 2005.
3. Hancock BW, Nazir K and Everard JE: Persistent gestational trophoblastic neoplasia after partial hydatidiform mole incidence and outcome. J Reprod Med 51: 764-766, 2006.

4. Shi YF, Li JQ, Zheng W, Chen XJ, Qiao YH, Hao M, Zhou CW, Hu YL, Wan GM, Sha YC and Zheng X: Survey of gestational trophoblastic disease incidence among 3.6 million pregnancies in China. Zhonghua Fu Chan Ke Za Zhi 40: 76-78, 2005 (In Chinese).

5. Sha YC: Investigation of Gestational trophoblastic disease in Anhui Province during 1991-2000. Anhui Medical Journal 3: 253-255, 2004 (In Chinese).

6. Song HZ, Yang XY and Xiang Y: Forty-five year's experience of the treatment of choriocarcinoma and invasive mole. Int $\mathrm{J}$ Gynaecol Obstet 60 (Suppl 1): S77-S83, 1998.

7. Feng FZ, Xiang Y, Shan Y, Wan XR and Oang XY: Clinical analysis of patients with lung metastasis of invasive mole before evacuation of hydatidiform mole. Zhonghua Fu Chan Ke Za Zhi 42: 830-833, 2007 (In Chinese).

8. Makangee A, Nadvi SS and Van Dellen JR: Invasive mole presenting as a spinal extradural tumor: Case report. Neurosurgery 38: 191-193, 1996.

9. Malhotra B and Misra R: Metastatic invasive mole in the urinary bladder. Indian J Cancer 39: 116-118, 2002.

10. Ngan HY, Bender H, Benedet JL, Jones H, Montruccoli GC and Pecorelli S; FIGO Committee on Gynecologic Oncology: Gestational trophoblastic neoplasia, FIGO 2000 staging and classification. Int J Gynaecol Obstet 83 (Suppl 1): S175-S177, 2003.

11. Lurain JR: Gestational trophoblastic disease I: Epidemiology, pathology, clinical presentation and diagnosis of gestational trophoblastic disease, and management of hydatidiform mole. Am J Obstet Gynecol 203: 531-539, 2010.

12. Chapman-Davis E, Hoekstra AV, Rademaker AW, Schink JC and Lurain JR: Treatment of nonmetastatic and metastatic low-risk gestational trophoblastic neoplasia: Factors associated with resistance to single-agent methotrexate chemotherapy. Gynecol Oncol 125: 572-575, 2012.

13. Yarandi F, Eftekhar Z, Shojaei H, Kanani S, Sharifi A and Hanjani P: Pulse methotrexate versus pulse actinomycin D in the treatment of low-risk gestational trophoblastic neoplasia. Int J Gynaecol Obstet 103: 33-37, 2008.

14. Escobar PF, Lurain JR, Singh DK, Bozorgi K and Fishman DA: Treatment of high-risk gestational trophoblastic neoplasia with etoposide, methotrexate, actinomycin D, cyclophosphamide, and vincristine chemotherapy. Gynecol Oncol 91: 552-557, 2003.

15. Lu WG, Ye F, Shen YM, Fu YF, Chen HZ, Wan XY and Xie X: EMA-CO chemotherapy for high-risk gestational trophoblastic neoplasia: A clinical analysis of 54 patients. Int J Gynecol Cancer 18: 357-362, 2008.

16. Cagayan MS: High-risk metastatic gestational trophoblastic neoplasia. Primary management with EMA-CO (etoposide, methotrexate, actinomycin D, cyclophosphamide and vincristine) chemotherapy. J Reprod Med 57: 231-236, 2012.

17. Neubauer NL, Latif N, Kalakota K, Marymont M, Small W Jr, Schink JC and Lurain JR: Brain metastasis in gestational trophoblastic neoplasia: An update. J Reprod Med 57: 288-292, 2012.

18. Soper JT, Spillman M, Sampson JH, Kirkpatrick JP, Wolf JK and Clarke-Pearson DL: High-risk gestational trophoblastic neoplasia with brain metastases: Individualized multidisciplinary therapy in the management of four patients. Gynecol Oncol 104: 691-694, 2007.

19. Newman LB, Morgan TE, Bucy JG and Wise L: Choriocarcinoma and bilateral renal metastases. Urology 5: 658-661, 1975.

20. Wang YE, Song HZ, Yang XY, Dong SY and Gan N: Renal metastases of choriocarcinoma. A clinicopathological study of 31 cases. Chin Med J (Engl) 104: 716-720, 1991. 